\title{
Belgeo
}

\section{Avec Marc Pain, voyage dans le temps chez les Lele du Kasai}

Marc Pain (2016), « Kasaï. Rencontre avec le roi des Lele », Carnets de voyage 1980-1981, préface de Viviane Baeke, Husson éditeur, Bruxelles, $143 \mathrm{p}$.

\section{Henri Nicolaï}

\section{(2) OpenEdition}

1 Journals

Édition électronique

URL : http://journals.openedition.org/belgeo/19591

DOI : 10.4000/belgeo.19591

ISSN : 2294-9135

Éditeur :

National Committee of Geography of Belgium, Société Royale Belge de Géographie

Référence électronique

Henri Nicolaï, « Avec Marc Pain, voyage dans le temps chez les Lele du Kasai », Belgeo [En ligne], 1 |

2017, mis en ligne le 31 mars 2017, consulté le 24 septembre 2020. URL : http://

journals.openedition.org/belgeo/19591; DOI : https://doi.org/10.4000/belgeo.19591

Ce document a été généré automatiquement le 24 septembre 2020.

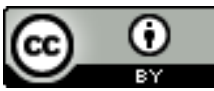

Belgeo est mis à disposition selon les termes de la licence Creative Commons Attribution 4.0 International. 


\section{Avec Marc Pain, voyage dans le temps chez les Lele du Kasai}

Marc Pain (2016), « Kasaï. Rencontre avec le roi des Lele », Carnets de voyage 1980-1981, préface de Viviane Baeke, Husson éditeur, Bruxelles, $143 \mathrm{p}$.

\section{Henri Nicolaï}

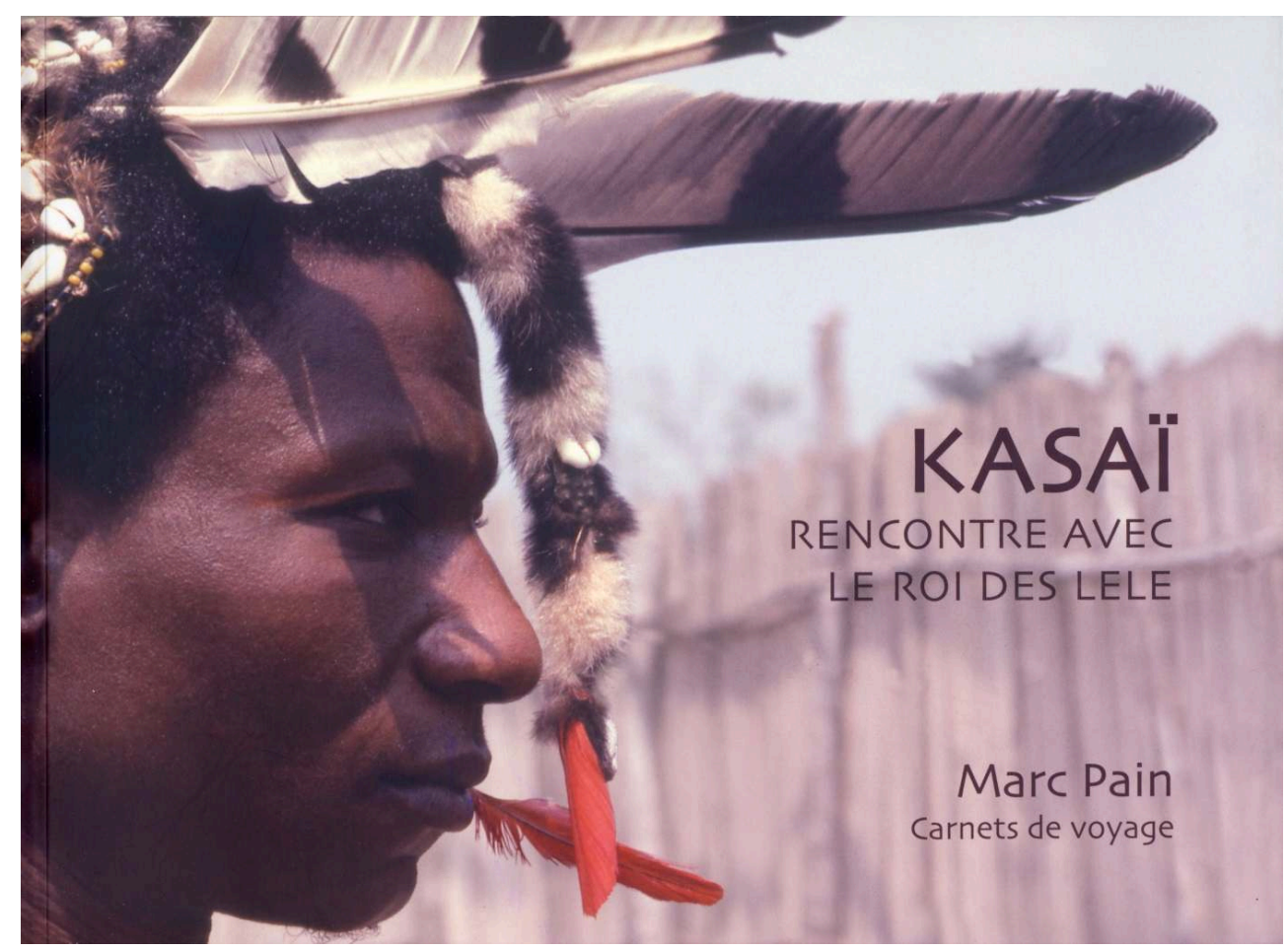

1 Marc Pain, dont on connaît la part prise à la conception et à l'élaboration de l'Atlas de Kinshasa, et aussi le livre tiré de sa thèse Kinshasa, la ville et la Cité (Orstom, 1984) et encore la participation à de grandes recherches multidisciplinaires sur la transmigration indonésienne, a eu l'heureuse idée de publier une sélection de photographies qu'il avait rapportées en 1980 et 1981 de deux séjours en pays lele, dans 
le Kasai occidental (l'auteur utilise la graphie Kasaï pour rappeler que son récit se place à l'époque du Zaïre). De belles photos dans une magnifique mise en page nous fournissent ainsi un témoignage exceptionnel sur une civilisation disparaissante et sur un de ses derniers sursauts.

Certes on peut soutenir que l'Afrique de l'avenir est celle des villes. Mais on ne peut oublier que la majorité de sa population vit encore dans les campagnes où elle combine les comportements de deux mondes, l'un hérité du passé traditionnel et l'autre engagé dans la société d'aujourd'hui.

Cette vie villageoise, nous l'avons nous-même évoquée récemment en mettant en ligne, d'une part sur le site de la Société royale belge de Géographie et d'autre part dans la rubrique Dossiers et archives de la revue BELGEO (2013) quelques photos prises de 1955 à 1958, donc à la fin de l'époque coloniale. Les photos de Marc Pain se placent un quart de siècle plus tard, vingt ans après l'accès du Congo à l'indépendance. Bien que les deux sélections soient de tailles très différentes, une centaine pour nos photos, presque le double pour celles de Marc Pain, le lecteur ne manquera pas de relever de nombreuses similitudes. C'est que les groupes concernés sont voisins. Nos photos se rapportent à des villages mbala ou pende, qui vivent à l'ouest des Lele.

Le groupe lele vit en territoire d'Ilebo, dans le Kasai occidental. A l'époque où Marc Pain l'a visité, il devait compter une quarantaine de milliers de personnes. Donc un groupe dont la taille était du même ordre de grandeur que le groupe voisin, les Kuba, qui se trouve à l'est de la rivière Kasai. Mais c'est huit fois moins que les Pende, ses voisins du sud-ouest. Les Lele ont été depuis longtemps relativement bien connus des ethnographes. Sous le nom de Bashilele, ils étaient réputés pour certaines institutions particulières dont nous reparlerons plus loin. Ils faisaient partie de cet ensemble de royaumes qui jalonnent la bordure savanisée australe de la forêt équatoriale. L'anthropologue anglaise Mary Douglas leur a consacré de nombreuses publications au cours de la dernière décennie du Congo colonial et a publié un livre tiré de sa thèse (The Lele of the Kasai, Oxford University Press, 1963). Dans plusieurs de ses articles, elle compare les institutions lele et celles des Kuba notamment dans leurs conséquences économiques. Nous avions utilisé ses conclusions dans un essai de géographie comparée de quatre groupes vivant sur le même parallèle, à savoir les Yaka, les Pende, les Lele et les Kuba en nous demandant notamment si les différences de densité de population, qui existent entre ces groupes, pouvaient s'expliquer par des différences entre les institutions (Quatre groupes humains du Congo méridional. Essai de géographie comparée, Mélanges de géographie... offerts à M. Omer Tulippe, I, 1967, p. 571-583). Mary Douglas avait montré notamment pour deux de ces groupes, les Lele et les Kuba, qu'ils avaient des institutions communes, qu'ils constituaient des royautés sacrées avec à la tête un Nyimi, mais que le groupe Kuba avait poussé beaucoup plus loin l'organisation de l'État. Le Nyimi des Lele est le chef du clan Tundu comme le Nyimi kuba est celui des Bushong. Mais alors que le tribut perçu par le Nyimi kuba est sans réciprocité (sauf la protection accordée au vassal), celui perçu par le Nyimi lele exige de sa part un don important, par exemple une épouse. Chez les Lele, une structure de subordination naissante a été neutralisée par son insertion dans une structure de réciprocité matrimoniale (L. de Heusch, Structures et praxis sociales chez les Lele du Kasai, L'Homme, IV, 3, 1964). En somme, les Kuba ont réussi le passage du clan à l'État. Les Lele n'ont pas dépassé celui du clan au village. Mary Douglas estime que l'on peut définir son ouvrage sur les Lele comme une étude sur l'absence d'autorité. 
D'autres aspects différencient la société lele de la société kuba. Les Kuba sont avides d'honneurs et de pouvoirs mais ceux-ci ne sont accessibles que par la richesse car les charges conférées par le roi exigent des dépenses importantes. Il y a là un stimulant efficace pour l'activité économique.

Chez les Lele, prestige, pouvoirs et honneurs s'obtiennent par l'âge. La société lele est une gérontocratie. Le pouvoir appartient à la classe des hommes âgés qui contrôle la classe des jeunes hommes. Les hommes âgés détiennent les femmes (ils sont généralement polygames) et les créances de femmes. C'est à eux que les jeunes hommes doivent, pendant des années, fournir de lourdes prestations, en produits agricoles, en travail, en monnaie traditionnelle (des tissus de raphia) pour pouvoir obtenir une femme en mariage. Il en résulte que les jeunes hommes ne peuvent se marier avant l'âge de trente-cinq ans. Pour tempérer ce système, la classe des hommes âgés fournit au groupe des jeunes hommes atteignant l'âge de dix-huit ans, une femme collective, au statut bien défini. C'est cette pratique qui avait valu aux Lele, dans la littérature ethnographique, la réputation d'être "polyandres ». Les jeunes hommes participaient fort peu aux activités économiques et dépensaient leur énergie dans des activités de chasse, dans les luttes qui opposaient jadis les villages et parfois dans des raids qui leur permettaient d'enlever des jeunes filles dans des villages éloignés.

7 Les institutions traditionnelles se sont maintenues chez les Kuba malgré la grave blessure d'amour-propre qu'a été, pour eux, l'acceptation du pouvoir colonial. La royauté notamment est restée bien vivante. Par contre, les institutions lele ont régressé. Lors de la visite de Marc Pain, la royauté était en déshérence depuis plus d'une génération, faute d'un accord entre les clans pour la désignation du Nyimi. Marc Pain a rencontré un jeune roi qui cherchait à rétablir le fonctionnement de cette royauté sacrée mais qui peinait à imposer son autorité. D'où son souci de se présenter à ses visiteurs avec l'apparat de sa cour et les attributs du pouvoir, vêtu, ainsi que ses principaux notables, de somptueux costumes et soucieux de faire respecter au plus près l'étiquette royale. Mais Pain a bien senti que son autorité était loin d'être établie.

8 Le pays lele venait d'ailleurs d'être ébranlé par un mouvement religieux, le culte mupele, qui s'attaquait à ses institutions traditionnelles. Mené tout particulièrement par un prêtre catholique local, s'appuyant sur des jeunes gens revenus de la ville, ce mouvement visait à éliminer les pratiques qu'il considérait comme relevant du monde démoniaque, visant tout particulièrement les guérisseurs, les gardiens des traditions orales et plus généralement les hommes âgés accusés de sorcellerie. Des pratiques d'exorcisme, dégénérant parfois en véritables tortures, avaient même entraîné plusieurs milliers de morts de même qu'elles avaient provoqué la destruction de nombreux objets rituels ou leur confiscation (parfois d'ailleurs suivie d'une vente à des trafiquants d'œuvres d'art). La gérontocratie lele a été affaiblie sinon même détruite. Ces circonstances, comme le souligne Viviane Baeke, du Musée de Tervuren, dans une pénétrante préface, confèrent au travail de Marc Pain, une valeur historique de premier plan car il se place à un moment essentiel de l'histoire contemporaine de la société lele.

9 Marc Pain présente ses photos dans le cadre d'un carnet de voyage. En effet, alors qu'il enseignait à Kinshasa, il avait été amené, pendant les vacances scolaires, à apporter son aide, principalement comme photographe et accessoirement comme chauffeur de son pick-up, à Mireille Alard, sa collègue de l'Institut Pédagogique National qui travaillait à 
une thèse sur l'art lele, thèse qu'elle soutiendra à l'Université de Paris 1 en 1987, sous le titre L'Art des Bashileele.

Le carnet, variante du récit de voyage, peut apparaître comme un genre un peu démodé mais il a ses charmes. Il met en contact direct le lecteur avec les paysages et les hommes. Les incidents anecdotiques qui parsèment le récit sont des instantanés spontanés qui nous informent en profondeur sur la vie quotidienne. Ils rendent compte aussi de l'atmosphère perçue par le voyageur

11 Et, dans le cas qui nous occupe, au sentiment d'abandon, de déclin, d'absence de perspectives et, en même temps, lorsque le contact s'établit plus en profondeur avec les villageois, l'impression malgré tout d'une société en mouvement. Les conversations fortuites ou organisées, par exemple le soir, autour du feu, révèlent, par bribes, les problèmes qui ont été évoqués plus haut.

12 Les deux premiers chapitres de l'ouvrage constituent le récit de voyage proprement dit avec la description de l'itinéraire de Kinshasa à Mapangu, pointe nord du pays lele (En camion sur la piste) et les déplacements Chez les Bashilele. Le délabrement de la route à l'est de Kikwit, les difficultés à trouver un véhicule, le recours parfois à la pirogue pour suppléer la panne ou l'encombrement d'un bac, sans compter les chicaneries ou les exigences de péage lors du franchissement de multiples barrages, ont fait de certains tronçons une aventure pénible. Le trajet, long d'un millier de kilomètres, a pris trois jours en 1980 et cinq jours en 1981. Le deuxième voyage est titré Retour et désillusions, car le sentiment de détérioration du pays lele a été ressenti davantage par les visiteurs, à l'exception d'une journée passée dans un village où une fête a été organisée avec des danses et avec la présentation de costumes d'apparat et de nombreux objets rituels de grande qualité esthétique.

On notera dans toute cette première partie une courte mais intéressante description des paysages des régions traversées, essentiellement le Kwango-Kwilu.

La deuxième moitié de l'ouvrage est une riche iconographie sur la vie villageoise (maisons, forges, métiers à tisser, etc.), sur les objets d'art et de tradition (grands tambours, costumes, coiffures, sculptures, tissus de raphia, oracles) et se termine par un gros chapitre sur les parades et les danses où le roi et sa cour apparaissent avec tout leur faste et où le lecteur est emporté par le mouvement des danses des femmes.

La collection photographique a été déposée au Musée royal de l'Afrique centrale à Tervuren. 